\title{
126. Sur les Algèbres de Lukasiewicz Injectives
}

\author{
Par Luiz MonTeIRo \\ Institut de Matemática Universidad Nacional \\ del Sur, Bahia Blanca \\ (Comm. by Kinjirô KunuGI, M.J.A., Sept. 13, 1965)
}

A. Monteiro a proposée ${ }^{1)}$ de déterminer les algèbres de Lukasiewicz (trivalentes) qui sont injectives et il a conjecturé qu'elles doivent être les algèbres complètes et centrées. Nous avons montré non seulement qu'il en est ainsi, mais aussi que ce résultat peut être considéré comme une conséquence d'un important théorème de Roman Sikorski [8].

Nous supposerons connues certaines notions sur les algèbres de Boole et sur les algèbres de Lukasiewicz (voir [3]-[7]).

Les opérateurs de possibilité $(M)$, de nécessité $(\nu)$ et de négation $(N)$ seront representés dans cette note respectivement par $\nabla, \Delta, \sim$.

Un centre d'une algèbre de Lukasiewicz $A$ est un élément $c \in A$ tel que $\sim c=c .^{2)}$ D'après Moisil $A$ ne peut avoir qu'un seul centre.

Si $A$ a un centre $c$, Moisil [5] a montré que pour tout $x \in A$ : $x=\Delta x \vee(c \wedge \nabla x \wedge \nabla \sim x)$.

Remarquons que

$$
x=(\Delta x \vee c) \wedge(\Delta x \vee \nabla x) \wedge(\Delta x \vee \nabla \sim x)
$$

et comme $\Delta x \vee \nabla x=\nabla x ; \Delta x \vee \nabla \sim x=\Delta x \vee \sim \Delta x=1$, alors on peut écrire plus simplement:

ou ce qui est équivalent

$$
x=(\Delta x \vee c) \wedge \nabla x
$$

$$
x=(\nabla x \wedge c) \vee \Delta x
$$

Un élément $k \in A$ sera dit booléen si $\dot{k}$ a un complément, c'està-dire s'il existe $k^{\prime} \in A$ tel que $k \vee k^{\prime}=1, k \wedge k^{\prime}=0$. S'il en est ainsi on a $k^{\prime}=\sim k$. D'après Moisil [3] pour qu'un élément $x$ soit booléen il faut et il suffit que $\nabla x=x$, ou ce qui revient au même $\Delta x=x$.

Représentons par $K(A)$ l'algèbre de Boole des éléments booléens de l'algèbre de Lukasiewicz $A$.

1.1. LEMME: Si C est une algèbre de Lukasiewicz complète, ${ }^{3)}$ alors l'algèbre de Boole $K(C)$ est complète.

Demonstration. Soit $\left\{k_{i}\right\}_{i \in I}$ une famille d'éléments de $K(C)$, et soit $k=\bigvee_{i \in I} k_{i}$. Montrons que $k \in K(C)$. En effet comme $k_{i} \leq k$ pour tout $i \in I$, alors, $\Delta$ étant monotone, nous pouvons écrire:

1) Dans un cour sur les Algèbres de Lukasiewicz réalisé pendant le premier semestre de 1963 à l'Universidad Nacional del Sur.

2) Voir [3] p. 446.

3) C'est-à-dire le réticulé $C$ est complet. 


$$
k_{i}=\Delta k_{i} \leq \Delta k \text { pour tout } i \in I
$$

d'oú $k=\bigvee_{i \in I} k_{i} \leq \Delta k \leq k$, donc $\Delta k=k$, et par conséquent $k \in K(C)$.

1.2. DEFINITION: Une algèbre de Lukasiewicz $C$ sera dite injective si quel que soit l'algèbre de Lukasiewicz $A$ et une sous algèbre $B$ de $A$, alors pour tout homomorphisme $f$ de $B$ dans $C$, il existe un homomorphisme $h$ de $A$ dans $C$ qui est une extension de $f\left(c^{\prime}\right.$ est-à-dire $h(b)=f(b)$ pour tout $\left.b \in B\right)$.

Il est bien connu que:

1.3. THEOREME: Toute algèbre de Lukasiewicz est sousalgèbre d'une algèbre de Lukasiewicz complète et centrée.

1.4. THEOREME: Une algèbre de Lukasiewicz $C$ est injective si et seulement si $C$ est complète et centrèe.

Demonstration. Nécessaire: Soit $C$ une algèbre de Lukasiewicz injective, alors, par le théorème 1.3. nous pouvons affirmer que $C$ peut être étendue à une algèbre de Lukasiewicz $A$ complète et centrée. Soit $f$ la transformation de la sous-algèbre $C$ de $A$ dans $C$, donnée par $f(x)=x$ pour tout $x \in C$. Il est évident que $f$ est un isomorphisme de $C$ sur $C$.

Comme $C$ est injective, alors il existe un homomorphisme $h$ de $A$ dans $C$ qui prolonge $f$.

Soit $\left\{c_{i}\right\}_{i \in I}$ une famille d'éléments de $C$. Montrons que cette famille a une borne supérieure dans $C$. En effet comme $c_{i} \in A$ pour tout $i \in I$ alors il existe l'élément $\bigvee_{i \in I} c_{i}=c_{0} \in A$, donc $c_{i} \wedge c_{0}=c_{i}$ pour tout $i \in I$, et par conséquent $h\left(c_{i} \wedge c_{0}\right)=h\left(c_{i}\right)$ c'est-à-dire $h\left(c_{i}\right) \wedge h\left(c_{0}\right)=$ $h\left(c_{i}\right)$. Comme $c_{i} \in C$ et $h$ est une extension de l'homomorphisme $f$, nous aurons $f\left(c_{i}\right) \wedge h\left(c_{0}\right)=f\left(c_{i}\right)$ pour tout $i \in I$, c'est-à-dire $c_{i} \wedge h\left(c_{0}\right)=$ $c_{i}$ pour tout $i \in I$, alors: $h\left(c_{0}\right) \in C$ est un majorant (dans $C$ ) de la famille $\left\{c_{i}\right\}_{i \in I}$.

Supposons maintenant qu'il existe un élément $c^{\prime} \in C$ tel que $c_{i} \leq c^{\prime}$ pour tout $i \in I$, alors $c_{0} \leq c^{\prime}$ et par conséquent $h\left(c_{0}\right) \leq h\left(c^{\prime}\right)=c^{\prime}$. Nous venons de montrer que $h\left(c_{0}\right) \in C$ est la borne supérieure (dans $C$ ) de la famille $\left\{c_{i}\right\}_{i \in I}$, donc $C$ est complète.

Voyons que $C$ est centrée. En effet comme $A$ a un centre $c$, alors nous aurons $h(c)=h(\sim c)=\sim h(c)$, donc $h(c)$ est un centre de $C$.

Suffisante: Soit $C$ une algèbre de Lukasiewicz complète et centrée, $A$ une algèbre de Lukasiewicz, $B$ une sous-algèbre de $A$ et $f$ un homomorphisme de $B$ dans $C$.

$K(A), K(C)$, et $K(B)$ sont des algèbres de Boole, et par le lemme 1.1., $K(C)$ est complète. Soit $f^{\prime}$ la restriction de $f$ à $K(B)$, alors $f^{\prime}$ est un homomorphisme booléen de $K(B)$ dans $K(C)$, donc par un théorème de Sikorski ${ }^{11}$ il existe un homomorphisme booléen $h^{\prime}$ de

1) Voir [8] et [2]. 
$K(A)$ dans $K(C)$ qui est une extension de $f^{\prime}$.

Posons par définition: $h(x)=\left(h^{\prime}(\Delta x) \vee c\right) \wedge h^{\prime}(\nabla x)$ pour tout $x$ de A.

Voyons maintenant que $h$ est un homomorphisme de $A$ dans $C$. Pour cela on doit démontrer que:

H1) $h(x \wedge y)=h(x) \wedge h(y)$.

En effet

H2)

$$
\begin{aligned}
h(x \wedge y) & =\left(h^{\prime}(\Delta(x \wedge y)) \vee c\right) \wedge h^{\prime}(\nabla(x \wedge y)) \\
& =\left(h^{\prime}(\Delta x \wedge \Delta y) \vee c\right) \wedge h^{\prime}(\nabla x \wedge \nabla y) \\
& =\left(\left(h^{\prime}(\Delta x) \wedge h^{\prime}(\Delta y)\right) \vee c\right) \wedge h^{\prime}(\nabla x) \wedge h^{\prime}(\nabla y) \\
& =\left(\left(h^{\prime}(\Delta x) \vee c\right) \wedge\left(h^{\prime}(\Delta y) \vee c\right)\right) \wedge h^{\prime}(\nabla x) \wedge h^{\prime}(\nabla y) \\
& =h(x) \wedge h(y) .
\end{aligned}
$$

$\sim h(x)=h(\sim x)$

$$
\begin{aligned}
\sim h(x) & =\sim\left(\left(h^{\prime}(\Delta x) \vee c\right) \wedge h^{\prime}(\nabla x)\right) \\
& =\left(\sim h^{\prime}(\Delta x) \wedge \sim c\right) \vee \sim h^{\prime}(\nabla x)
\end{aligned}
$$$$
=\left(\left(h^{\prime}(\sim \Delta x) \wedge c\right) \vee h^{\prime}(\sim \nabla x)\right.
$$$$
=\left(h^{\prime}(\nabla \sim x) \vee h^{\prime}(\Delta \sim x)\right) \wedge\left(c \vee h^{\prime}(\Delta \sim x)\right)
$$$$
=\left(h^{\prime}(\Delta \sim x) \vee c\right) \wedge\left(h^{\prime}(\nabla \sim x \vee \Delta \sim x)\right)
$$$$
=\left(h^{\prime}(\Delta \sim x) \vee c\right) \wedge h^{\prime}(\nabla \sim x)
$$$$
=h(\sim x) \text {. }
$$

H3) $\quad \nabla h(x)=h(\nabla x)$.

$$
\begin{aligned}
h(x) & =\nabla\left(h^{\prime}(\nabla x) \wedge\left(h^{\prime}(\Delta x) \vee c\right)\right) \\
& =\nabla h^{\prime}(\nabla x) \wedge\left(\nabla h^{\prime}(\Delta x) \vee \nabla c\right) \\
& =\nabla h^{\prime}(\nabla x) \wedge\left(\nabla h^{\prime}(\Delta x) \vee 1\right) \\
& =\nabla h^{\prime}(\nabla x)=h^{\prime}(\nabla x) \\
& =h^{\prime}(\nabla x) \wedge\left(h^{\prime}(\nabla x) \vee c\right) \\
& =h^{\prime}(\nabla \nabla x) \wedge\left(h^{\prime}(\Delta \nabla x) \vee c\right)=h(\nabla x) .
\end{aligned}
$$

Voyons finalement que $h$ est une extension de $f$.

Soit $b \in B$, alors comme $B$ est une sous-algèbre de $A$, nous aurons $\Delta b, \nabla b \in B$, plus précisément $\Delta b, \nabla b \in K(B)$, donc:

(i) $\quad h^{\prime}(\Delta b)=f^{\prime}(\Delta b)=f(\Delta b)$,

(ii) $\quad h^{\prime}(\nabla b)=f^{\prime}(\nabla b)=f(\nabla b)$

et par ailleurs nous pouvons écrire, en utilisant (i) et (ii):

$$
\begin{aligned}
h(b) & =h^{\prime}(\nabla b) \wedge\left(h^{\prime}(\Delta b) \vee c\right)=f(\nabla b) \wedge(f(\Delta b) \vee c) \\
& =\nabla f(b) \wedge(\Delta f(b) \vee c)=f(b)
\end{aligned}
$$

et la démostration est terminée.

\section{Bibliographie}

[1] G. Birkhoff: Lattice Theory. Revised Edition (1948).

[2] P. Halmos: Injective and Projective Boolean Algebras. Proc. of Symposia in Pure Math., 2, 114-122 (1961).

[3] G. Moisil: Recherches sur les logiques non-chrysipiennes. Annales Scientifiques de l'Université de Jassy, 26, 431-466 (1940). 
[4] - : Notes sur les logiques non-chrysipiennes. Annales Scientifiques de l'Université de Jassy, 27, 86-98 (1941).

[5] - - Sur les anneaux de caractéristique 2 ou 3 et leurs applications. Bulletin de l'Ecole Polytechnique de Bucarest, 12, 66-90 (1941).

[6] A. Monteiro: Sur la définition des algèbres de Lukasiewicz trivalentes. (A paraître dans le Bulletin de la Société des sciences mathématiques de la R. P. Roumaine)

[7] L. Monteiro: Axiomes indépendants pour les algèbres de Lukasiewicz trivalentes. (A paraître dans le Bulletin de la Société des sciences mathématiques de la R. P. Roumaine)

[8] R. Sikorski: A theorem on extensions of homomorphisms. Annales de la Société Polonaise de Mathématiques, 21, 332-335 (1948). 